\title{
In vivo studies of resveratrol contained in cosmetic emulsions
} \begin{abstract}
Poznań, Poland
The cosmetic and pharmaceutical industry is constantly on the lookout for new and efficient active substances. In this article, we focus on resveratrol, an active substance with beneficial health properties, which is obtained, among others, from vines and knotweed (Reynoutria japonica). In this study, we assessed the level of skin hydration in a group of probands who used cream with or without resveratrol. The compound has been shown to have moisturizing and tightening properties, improving the overall condition of the skin.
\end{abstract}

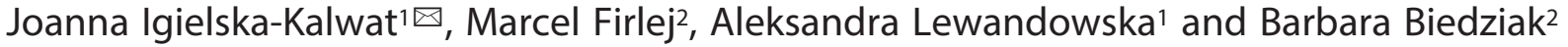

${ }^{1}$ Higher School of Health, Beauty and Education, Poznań, Poland; ${ }^{2}$ Department of Facial Malformations, Poznan University of Medical Sciences,

Key words: resveratrol, moisturizing, skin, cosmetic formulations hydration.

Received: 17 June, 2019; revised: 17 July, 2019; accepted: 17 July, 2019; available on-line: 12 September, 2019

๑e-mail: ji11602@amu.edu.pl

Abbreviations: a.u., contractual units; cold $\mathrm{O} / \mathrm{W}$ cream, $p, p$-value UV, ultraviolet

\section{INTRODUCTION}

The cosmetics industry has now become one of the most dynamically developing industries. The development of civilization and technology forces producers to research and introduce new cosmetic forms. There is a noticeable increase in consumer awareness as well as the impact of the ecological trend; hence, manufacturers are obliged to look for new active substances that will meet consumers' expectations (Burns et al., 2002). Biologically active substances are used in the production of many cosmetic or pharmaceutical formulations and dietary supplements. They are supposed to lead to more effective operation of cosmetic or pharmaceutical formulations. All research institutes are looking for biologically active forms that will contain effective ingredients in the fight against skin problems, delaying the aging process. One of the substances of interest is resveratrol (phytoalexin), a phenolic compound derived from vines, eucalyptus, knotweed, spruce, mulberry, lilies and peanuts (Burns et al., 2002). This stilbenoid belongs to the group of plantderived flavonoids. In wine and grapes, it occurs in the biocompartment containing procyanidins, anthocyanins and flavonols. The average resveratrol content in red wines is $1.9 \mathrm{mg} / \mathrm{dm}^{3}$. Resveratrol is a phytoestrogen, i.e., a plant hormone with an estrogen-like effect. This compound occurs in two forms, cis- and trans- (Anisimova et al., 2011). The trans form can be found in plants (phenol stilbene), while the cis form is formed by isomerization of trans-resveratrol and as a result of the disintegration of the resveratrol polymer molecule during the fermentation of grape skins with the participation of UV rays and a high $\mathrm{pH}$ value (Kalantari et al., 2010). Transresveratrol is characterized by a higher biological activity compared to the cis- form. The trans form of resveratrol is much better researched than the cis- formone (Fig. 1) (Orallo, 2006).

The interest in resveratrol increased with the observation of the "French paradox", as analysed in detail by Roger Corder. The researcher explains why the French enjoy good health and rarely suffer from heart diseases despite the diet rich in fat and little physical activity. Corder claims it is because they consume red wine with each meal. Studies have shown that white and rosé wine are not characterised by as high biological activity as red wine (Corder, 2008). Resveratrol together with polyphenols neutralize harmful fatty acids, lower the cholesterol levels, have anti-inflammatory properties and are effective antioxidants. The consumption or application of resveratrol causes the increase of lipoprotein blood level, complexes which are responsible for inhibiting the development of atherosclerosis. They also intensify the breakdown of fibrin protein, which is precipitated during blood coagulation as a result of which the function of platelets slows down. Both of the above described action limit the risk of coronary heart disease (Keylor et al., 2015).

Further, resveratrol slows skin aging. As the cells get older, their genetic material is damaged. This affects proper operation of sirtuins, enzyme proteins discovered at the beginning of the 21st century. Their function in the body is responsibility for regeneration, vitality and resistance of cells. While their activity decreases with age, resveratrol is one of the best sirtuin activators. It affects skin repair processes, prolongs the life of skin cells and protects against adverse effects of external factors such as UV radiation. Another process of destroying skin support proteins, i.e. collagen and elastin, by sugars is referred to as glycation, particularly prevalent in people with diabetes and the elderly. There is a decrease in the regeneration ability and more frequent occurrence of inflammation. The anti-glycation effect that resveratrol possesses is the protection of collagen and elastin from destruction and disorganization (Zhou et al., 2007). Finally, free radicals are reactive molecules formed under the influence of, among others, UV radiation, toxins, tobacco smoke and stress. They affect the skin negatively, accelerating premature aging. With age, the system of



cis-Resveratrol

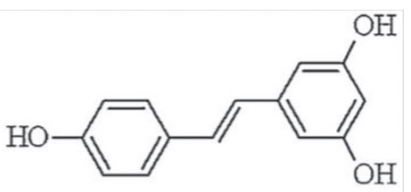

trans-Resveratrol
Figure 1. The chemical structure of resveratrol (cis and trans forms). 
protection against free radicals is weakened. Resveratrol is one of the most effective antioxidants, as it protects the skin against free radicals and delays the aging process. It also acts as a whitening agent for the skin, by inhibiting the activation of the tyrosinase enzyme which is involved in the production of skin pigments ( $\mathrm{Li}$ et al., 2016). The discussed compound has a positive effect on bursting blood vessels, reduces swelling and redness. It also accelerates the wound healing process and has soothing properties. Further, the component improves the hydration levels and structure of the skin, because it affects the production of glycosaminoglycans responsible for the hydration of the skin. It is also recommended for women during menopause who may have problems with regeneration, thickness and structure of their skin. An important property of resveratrol is the anti-wrinkle action consisting in reducing existing mimic wrinkles (Marques et al., 2009).

\section{MATERIALS AND METHODS}

The study was performed in a group of 20 volunteers over a period of 6 weeks. Each participant received one of two creams, one with resveratrol or the other with the same base but without resveratrol (placebo cream). The volunteers tested cosmetic products, applying them on the face twice a day, in the morning and in the evening. Prior to the tests, the skin of the probands was examined in order to diagnose its condition (set as the zero state). Then, subsequent digital measurements were made every 2 weeks. Physical studies were conducted using the Courage+Khazaka Multi Skin Test Center ${ }^{\circledR}$ MC 1000 device. The hydration of the epidermis was evaluated with a corneometer which measures the hydration level of the superficial layers of the skin (stratum corneum), based on electrical conductivity. The higher the

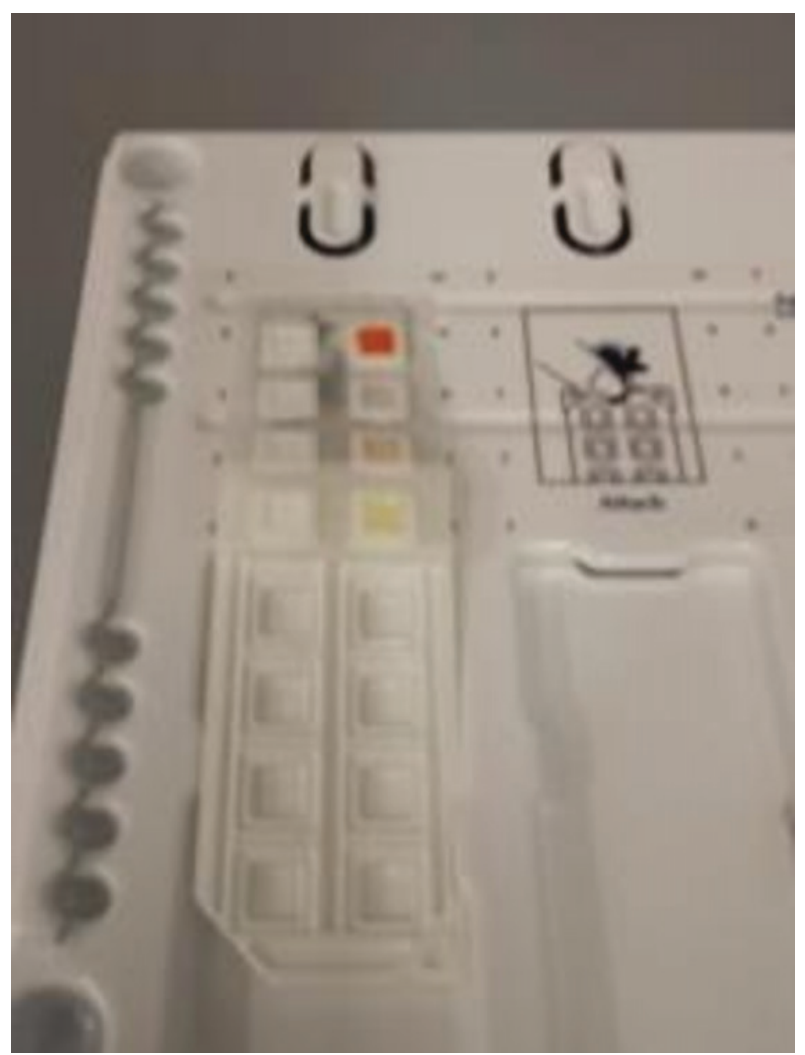

Figure 2. The T.R.U.E. TEST prepared for application. water content in the epidermis, the smaller the capacitive resistance, which indicates a higher degree of skin hydration.

Before starting the analysis, every volunteer underwent T.R.U.E. TEST, to diagnose potential allergic contact dermatitis. The T.R.U.E. TEST is a hypoallergenic test done with water-proof patches. Resveratrol was put on the patches and applied to the skin of the back of the volunteers, for 48 hours. At that time, the subjects were asked not to have excessive physical activity, to avoid the sun and to not take steroid drugs. After this time, the backs of the probands were checked using the IQ Ultra Reading Plate. After 96 hours, the skin of the participants was checked again (as per test instructions of the IQ Ultra test from Chemotechnique Diagnostics) (User Manual. Chemotechnique Diagnostics: IQ Ultra).

In all 20 probands, the test results were negative. There was no visible reaction, meaning that they did not show an allergic response to resveratrol (Fig. 2).

All the volunteers were asked to complete a questionnaire, which addressed their knowledge of the tested ingredient, their age, their awareness of complexion type they had and their possible allergic reactions. They were also asked to sign an informed consent to participate in the experiment. Before the test, an interview was conducted with each proband where the principles of product testing and the possible side effects were explained. This research was conducted with the permission of the Poznań Medical University Bioethical Commission. The volunteers received written instructions for using the cosmetic. During the tests, they could not use other cosmetics or a beautician's services. Thanks to the sensory evaluation, qualitative analysis and subjective assessment of the tested cosmetic, we were able to learn the opinion of potential consumers (Dębowska, 2010).

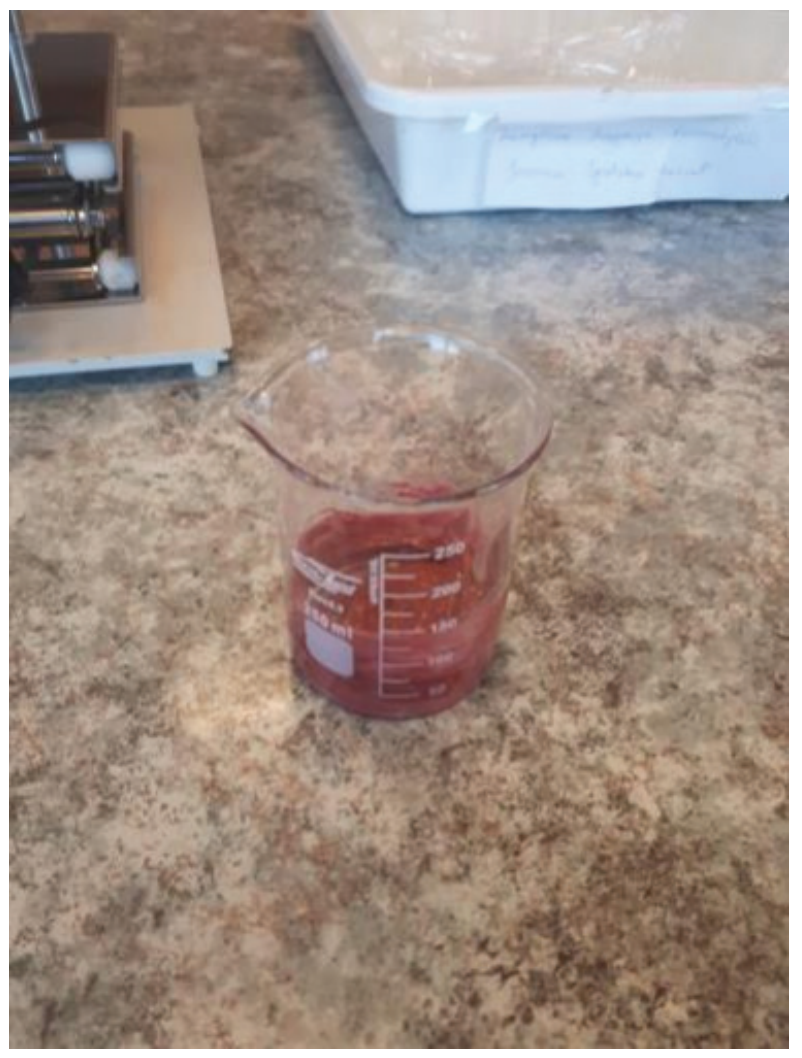

Figure 3. Cold O/W cream with resveratrol. 
Table 1. Ingredients of cold $\mathrm{O} / \mathrm{W}$ creams with resveratrol

\begin{tabular}{ll}
\hline Composition & Content $(\%+0.001) \mathrm{g}$ \\
\hline $\begin{array}{l}\text { Creagel EZ7 hydrogenated polydecene, } \\
\text { Lauryl polyoxyethylene ether }\end{array}$ & 5000 \\
\cline { 1 - 1 } $\begin{array}{l}\text { Alphaflow } 20 \text { hydrogenated polyde- } \\
\text { cene }\end{array}$ & 8000 \\
\hline $\begin{array}{l}\text { Distilled water } \\
\text { Resveratrol (F, Fesveratrol; OPC, For- } \\
\text { meds }\end{array}$ & 0.007 \\
\hline
\end{tabular}

\section{PREPARATION}

$5.000 \mathrm{~g}$ of Creagel and $8.000 \mathrm{~g}$ of Alphaflow (Table 1) were weighed in a $50 \mathrm{ml}$ beaker. $35 \mathrm{~g}$ of distilled water was weighed in another $50 \mathrm{ml}$ beaker. The contents of the first beaker were thoroughly mixed with a magnetic stirrer at room temperature while the distilled water was added in small portions. Next, $7 \mathrm{mg}$ of trans-resveratrol was added to the mix and stirring continued for 5 minutes. A thick pink-violet cream was obtained (Fig. 3) (Igielska-Kalwat, 2018).

The placebo (control) cream consisted only of the Creagel and Alphaflow base, with no active substance (resveratrol) added (as per the BN-64/6140-02 standard for cream quality control) (BN-64/6140-02 Standard Cream Quality Control).

\section{RESULTS AND DISCUSSION}

The purpose of moisturizing preparations is not to introduce water into the skin, but to limit its loss from the dermis. The most effective method for moisturizing the skin is to strengthen the barriers that inhibit water loss. The reduction of water evaporation from the epidermis is directly related to the increase in its hydration.

Figure 4 presents the average changes in the hydration level of the tested probands who applied cream with resveratrol to their skin.

The emulsions containing resveratrol significantly influenced the hydration level of the epidermis. The average hydration value, which was observed in probants before using emulsion, amounted to 35.00 a.u. After one week of application.

During the regular use of the preparation, the hydration level increased on average by 9 a.u. after the second

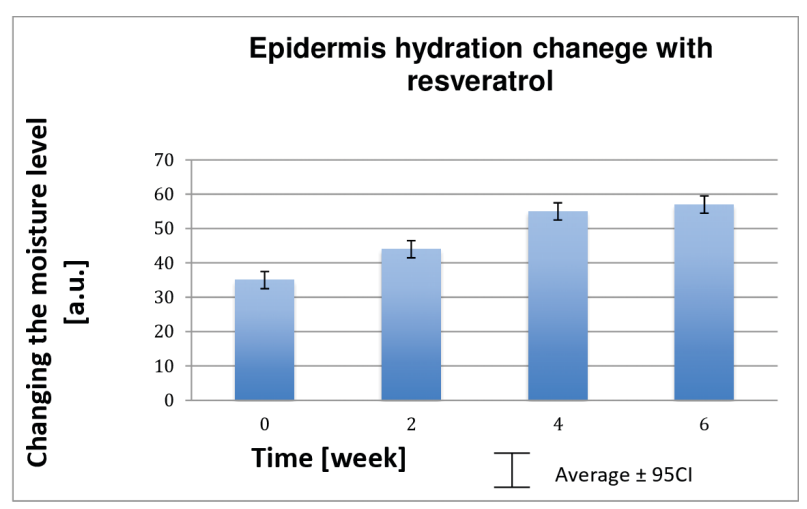

Figure 4. Hydration of the epidermis increases from the start of applying the formulation containing resveratrol to 6 weeks after regular use of the cosmetic.

${ }^{*} p<0.01$ as a significant improvement from week 0 to week 6 (W6), determined by the post-hoc test. Total trial group $(n=20)$. Measurements were taken every two weeks.
Epidermis hydration chanege without resveratrol

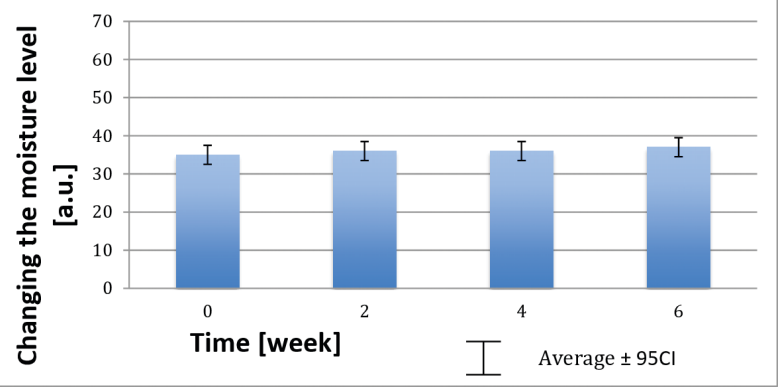

Figure 5. Hydration increases from the start of applying the control formulation without resveratrol and still after (how many?) weeks of using the cosmetic.

${ }^{* *} p<0.01$ as a significant improvement from week 0 to week 6 (W6), determined by the post-hoc test. Total trial group $(n=20)$. The measurements were taken every two weeks.

week of use. In the subsequent two weeks, an increase in hydration by $20 \%$ was observed in the test subjects. The measurements taken in the fourth week showed an increase in hydration by $201 \%$. After 6 weeks, the parameter increased by $3.5 \%$. As the active ingredient of the emulsion is hydrophobic, it creates a waterproof filter on the surface of the epidermis. It can be concluded that resveratrol penetrates the epidermis, thus supplementing the lipids of its protective layer. Therefore, during the use of the tested emulsion, the hydration level increased (Yang et al., 2017; Igielska-Kalwat J, Tariq Mahmood, 2018; Vries et al., 2018).

Figure 5 presents the average changes in the skin hydration level in the control group.

The results showed that the formula which did not contain resveratrol also increased hydration of stratum corneum, which is associated with the presence of lipid emulsions in the composition. They supplement or modify the natural water-lipid layer and intercellular cement. Therefore, they inhibit the transepidermal water loss (TEWL) by creating skin occlusion, and increase hydration of the epidermis by building in the cement lipids. The average hydration value, which was observed in probants before using emulsion, amounted to 35.00 a.u. After one week of application. After the second week, an increase of $2.7 \%$ in skin hydration was found, and the hydration level remained stable until the last week. After six weeks, an increase in hydration by $5.4 \%$ was noted, showing much lower effectiveness of the prepared emulsion compared to the one containing resveratrol. It can be concluded that the analyzed emulsion is a cosmetic that does not improve skin hydration as efficiently as a cream containing the active substance (Nawaz et al., 2018; Yang et al., 2015).

\section{CONCLUSION}

In the in vivo studies on volunteers using self-made cosmetic emulsions containing resveratrol, the tested cream proved to be an effective cosmetic that improved the functioning of the hydro-lipid barrier of the skin, increasing the level of skin hydration. Resveratrol did not show to be a sensitizer in the T.R.U.E TEST, i.e. the method for assessing irritation by dermatological evaluation. Based on the study, the compound was confirmed to have moisturizing and tightening properties that improve the general condition of the skin. 


\section{Acknowledgement}

Financial support from Polish Ministry of Science and Higher Education is acknowledged.

\section{Conflict of Interest}

None

\section{REFERENCES}

Anisimova NY, Kiselevsky MA, Sosnov AV, Sadovnikov SV, Stankov IN, Gakh AG (2011) Trans-, cis-, and dihydro-resveratrol: A comparative study. Chem Cen J 5: 88-92. https://doi.org/10.1186/1752153X05088

BN-64/6140-02 Standard - Cream Quality Control

Burns J, Yokota T, Ashihara H, Lean MEJ, Crozier A (2002) Plant foods and herbal sources of resveratrol. J Agric Food Chem 50: 33373340. https://doi.org/10.1021/jf0112973

Corder R (2008) The Wine Diet. Warszawa.

Vries DE, Strydom K, Steenkamp V (2018) Bioavailability of resveratrol: Possibilities for enhancement. J Herb Med 11: 1-77. https://doi. org $/ 10.3390 /$ biomedicines 6030091

Dębowska R (2010) In vitro and ex vivo tests in contemporary cosmetology. Chemike 2: 74-79

Igielska-Kalwat J (2018) Studies on stability of emulsion containing carotenoids. Acta Biochim Pol 3: 455-463. https://doi.org/10.18388/ abp.2018_2589

Igielska-Kalwat J, Tariq Mahmood (2018) In vivo research of cosmetic forms containing $\beta$-carotene. Acta Pol Pharma 75: 313-320. https:// doi.org/10.32383/appdr/86683

Kalantari H, Das Dipak K (2010) Physiological effects of resveratrol. Bio Factors 36: 401-406. https://doi.org/10.1002/biof.100
Keylor MH, Matsuura BS, Stephenson CRJ (2015) Chemistry and biology of resveratrol-derived natural products. Chem Rev 115: 89769027. https://doi.org/10.1021/cr500689b

Li M, Schneider K, Kristensen M, Borodina I, Nielsen J (2016) Engineering yeast for high-level production of stilbenoid antioxidants. Sci Rep 11: 36827. https://doi.org/10.1038/srep36827

Marques FZ, Markus MA, Morris BJ (2009) Resveratrol: Cellular actions of a potent natural chemical that confers a diversity of health benefits. Int J Biochem 41: 2125-2128. https://doi.org/10.1016/j. biocel.2009.06.003

Nawaz W, Zhou Z, Deng S, Ma X, Ma X, Li C. Shu, X (2018) Therapeutic versatility of resveratrol derivatives. Nutrients 9: 1188-1192. https://doi.org/10.3390/nu9111188

Orallo F (2006) Comparative studies of the antioxidant effects of cis- and trans-resveratrol. Curr Med Chem 13: 87-98. https://doi. org/10.2174/092986706775197962

User Manual. Chemotechnique Diagnostics: IQ Ultra.

Yang SC, Tseng CH, Wang PW, Lu PL, Weng YH, Yen FL, Fang JY (2017) Pterostilbene, a methoxylated resveratrol derivative, efficiently eradicates planktonic, biofilm, and intracellular MRSA by topical application. Front Microbiol 8: 1103-1110. https://doi.org/10.3389/ fmicb.2017.01103

Yang T, Wang L, Zhu M, Zhang L, Ln L (2015) Properties and molecular mechanisms of resveratrol: A review. Pharmazie 70: 501-506. https://doi.org/10.3389/fphar.2018.00444

Zhou J, Yang L, Wang C, Choi ES, Kim SW (2007) Enhanced performance of the methylerythritol phosphate pathway by manipulation of redox reactions relevant to IspC, IspG, and IspH. J Biotechnol 248: 1-8. https://doi.org/10.1016/j.jbiotec.2017.03.05 